\title{
Influência do teor de cerâmica vermelha do agregado reciclado nas propriedades do concreto permeável
}

\author{
Influence of red-clay ceramic content of \\ recycled aggregate on the properties of \\ pervious concrete
}

Rafael Jansen Mikami ${ }^{1}$, Patricia Kruger ${ }^{2}$, Eduardo Pereira ${ }^{2}$, Ana Carolina Barbosa Kummer', Maria Magdalena Ribas Döll ${ }^{2}$

\footnotetext{
${ }^{1}$ Universidade Estadual de Ponta Grossa (UEPG), Programa de Pós-Graduação em Engenharia Sanitária e Ambiental (PPGESA), Av. Carlos Cavalcanti, 4748, Uvaranas, Ponta Grossa, PR, Brasil. e-mail: rjmikami@outlook.com

${ }^{2}$ Universidade Estadual de Ponta Grossa (UEPG), Departamento de Engenharia Civil, Uvaranas, Ponta Grossa, PR, Brasil.

e-mail:pkruger@uepg.br; eduardopereira@uepg.br; ackummer@hotmail.com; mmrdoll@uepg.br
}

\section{RESUMO}

Os resíduos de construção e demolição podem ser utilizados como agregados reciclados em diferentes aplicações do setor da construção civil. A substituição do agregado natural do concreto permeável por agregados reciclados é uma alternativa que garante uma destinação final a esses materiais. No entanto, a composição dos resíduos irá influenciar diretamente nas propriedades do concreto permeável, principalmente no que se refere à quantidade de material cerâmico. Dessa forma, neste estudo foi analisada a influência da composição dos agregados reciclados na resistência e permeabilidade do concreto poroso. Para isso, agregados contendo teores de resíduo de cerâmica vermelha de 10, 25, 50 e 100\% foram obtidos pela mistura de um resíduo de construção e demolição (com 2,92\% de cerâmica) e resíduos de blocos cerâmicos. Os concretos permeáveis foram caracterizados conforme a resistência à compressão axial, coeficiente de permeabilidade, índice de volume de vazios, absorção de água e massa específica real e aparente. A permeabilidade de todos os concretos permeáveis variou entre 0,82 e $0,95 \mathrm{~cm} . \mathrm{s}^{-1}$. O coeficiente de permeabilidade reduziu com o aumento da quantidade de cerâmica na mistura. A resistência à compressão axial do concreto com $0 \%$ de cerâmica foi superior aos demais concretos, resultando em 10,15 MPa. Os concretos que continham cerâmica apresentaram resistência inferior a 3,00 $\mathrm{MPa}$. O volume de vazios dos concretos não variou significativamente, se situando entre 32,13 e $37,35 \%$. Não foi verificada relação direta entre o volume de vazios, permeabilidade e resistência à compressão. No entanto, a resistência foi maior com o aumento da massa específica aparente e o coeficiente de permeabilidade foi menor nos concretos de maior absorção de água.

Palavras-chave: Resíduo de construção e demolição, pavimento poroso, resistência, permeabilidade.

\begin{abstract}
Construction and demolition waste can be used as recycled aggregates in different civil construction applications. The substitution of natural aggregate from pervious concrete by recycled aggregates is a sustainable alternative that ensures a final destination of these materials. However, the composition of such waste will influence directly the properties of the pervious concrete, especially with regard to the amount of ceramic material. Thus, this study analyzed the influence of recycled aggregates composition on the strength and permeability of pervious concrete. Aggregates containing 10, 25, 50 and 100\% red-clay ceramic waste were obtained by mixing a construction and demolition waste (with $2,92 \%$ ceramic) and ceramic brick waste. A natural aggregate and a recycled concrete aggregate were also analyzed. The pervious concrete was characterized with regard to compressive strength, permeability coefficient, void content, water absorption and specific mass. The permeability of all pervious concretes ranged between 0,82 and $0,95 \mathrm{~cm} . \mathrm{s}^{-1}$. The permeability coefficient decreased with the increase in the amount of ceramic in the mixture. The compressive strength of
\end{abstract}


the concrete with $0 \%$ ceramic was higher than the other concretes, resulting in 10,15 MPa. The concretes containing ceramic resulted in strength below 3,00 MPa. The void content of the concretes did not vary significantly, ranging between 32,13 and $37,35 \%$. No direct relation was observed between the void content, permeability and compressive strength. However, the strength was higher with the apparent specific mass increase and the permeability coefficient was lower in the concretes with higher water absorption.

Keywords: Construction and demolition waste, porous pavement, strength, permeability.

\section{INTRODUÇÃO}

O uso de resíduos de construção e demolição (RCD) como agregados reciclados é uma prática que tem sido estudada amplamente nos últimos anos [1-6]. A reciclagem do RCD é um avanço para a sustentabilidade no setor da construção civil, visto que esse processo reduz a exploração de recursos naturais e propicia uma destinação final para parte dos resíduos gerados [3]. Segundo a Associação Brasileira de Empresas de Limpeza Pública e Resíduos Especiais (ABRELPE) [7], cerca de 45 milhões de toneladas de RCD foram gerados no Brasil em 2014, o que corresponde a aproximadamente 50\% em massa do total de resíduos sólidos gerado no ano. Devido a esse volume excessivo de resíduos, há uma preocupação em realizar a destinação correta dos materiais, sendo o gerenciamento do RCD regulamentado pela Resolução no 307 do CONAMA [8], de 2002.

As principais utilizações do agregado reciclado no setor da construção civil são o uso na pavimentação (como material de base e sub-base) e a confecção de concretos e argamassas [9]. De acordo com Rodríguez et al. [4], o reuso do RCD em países como Dinamarca, Estônia, Alemanha e Inglaterra chega a atingir até 75\%. De acordo com a Associação Brasileira para Reciclagem de Resíduos da Construção Civil e Demolição (ABRECON) [10], estima-se que em 2015 o Brasil possuía capacidade para reciclar até 46\% do volume de RCD gerado, no entanto a produção de agregados reciclados foi de apenas 21\%. A NBR 15116/2004 [11] limita a utilização de agregados reciclados a usos na pavimentação e preparo de concretos sem função estrutural, o que restringe a reutilização do RCD. Uma forma de ampliar o emprego dos resíduos de construção é realizar a sua incorporação no concreto permeável, reduzindo assim o consumo de agregados convencionais.

O concreto permeável, também denominado de concreto poroso, é produzido pela mistura de agregados graúdos, cimento e água, formando uma estrutura contendo vazios interligados [12-14]. O volume de vazios formado no concreto permeável varia de 15 a 35\% [13, 15,16], o que permite a passagem da água pelo seu interior. Essa característica possibilita a sua aplicação em diferentes utilizações, como pavimentos de tráfego leve, calçadas, estacionamentos e pisos externos [17]. Os pavimentos de concreto poroso são úteis para a gestão de águas pluviais, visto que reduzem o escoamento superficial da água, permitem a recarga de águas subterrâneas e são medidas que podem mitigar problemas de drenagem como as inundações, já evidentes em grandes centros urbanos [15].

A permeabilidade mínima de um pavimento permeável, de acordo com a NBR 16416/2015 [18], é de $0,10 \mathrm{~cm} \cdot \mathrm{s}^{-1}$. Os valores de coeficiente de permeabilidade usualmente variam de 0,20 a $0,54 \mathrm{~cm} \cdot \mathrm{s}^{-1}$ [15], porém valores superiores já foram encontrados em estudos recentes [19, 20, 21]. Estudos indicam que concretos com índice de vazios de $15 \%$ já atingem a permeabilidade mínima [13]. Uma maior quantidade de poros tenderia aumentar proporcionalmente a permeabilidade. Entretanto, essa propriedade não é afetada apenas pela quantidade de vazios no concreto, mas também pela sua distribuição e interligação [22].

A dosagem do concreto permeável tem como objetivo equilibrar as suas propriedades de resistência e permeabilidade [13]. No entanto, existem muitas dificuldades nesse processo, visto que essas propriedades dependem de diversos fatores, como a distribuição granulométrica, o tamanho e tipo do agregado, o grau de compactação da mistura, a relação água/cimento e o consumo de cimento [19, 22-25].

$\mathrm{O}$ volume de vazios do concreto poroso usualmente é associado a sua baixa resistência à compressão [26]. DEO e NEITHALATH [27] verificaram redução de até 50\% na resistência à compressão devido ao aumento em $10 \%$ do volume de vazios. TORRES et al. [19] verificaram que maiores quantidades de cimento resultaram em uma maior espessura da pasta de cimento que envolve os agregados, aumentando a resistência à compressão. Entretanto, isto reduziu o volume de vazios, sendo prejudicial à permeabilidade. A relação água/cimento também é um parâmetro importante para as características mecânicas, no entanto, as relações do concreto convencional não podem ser aplicadas. Pouca quantidade de água pode ocasionar uma falta de aderência entre o agregado e a pasta de cimento, além de problemas na trabalhabilidade [13]. Por outro lado, o excesso de água provoca o escoamento da pasta de cimento, que passa a preencher os vazios afetando a permeabilidade do concreto [15].

A substituição do agregado natural por resíduos de construção e demolição no concreto permeável afeta tanto a permeabilidade como a resistência do material. Em geral, os concretos permeáveis contendo 
RCD apresentam menor resistência, porém maior índice de vazios e permeabilidade [17,28]. No entanto, ZAETANG et al. [20] verificaram que a substituição parcial do agregado natural por agregado reciclado de concreto promoveu um aumento na resistência à compressão do concreto permeável.

Ainda são necessários estudos que abordem a influência da composição do agregado reciclado nas propriedades do concreto permeável, principalmente no que se refere ao teor de material cerâmico. Os resíduos de cerâmica vermelha são materiais porosos e de acordo com ANGULO et al. [29] isso reflete em prejuízos na resistência mecânica e durabilidade do concreto. Devido à falta de separação dos resíduos, o RCD pode apresentar teores de cerâmica vermelha prejudiciais às propriedades do concreto. Dessa forma, o objetivo deste estudo foi analisar concretos produzidos com agregados reciclados compostos por diferentes quantidades de cerâmica vermelha em sua composição e verificar o efeito na permeabilidade e resistência do concreto permeável.

\section{MATERIAIS E MÉTODOS}

Para avaliar a influência do teor de cerâmica vermelha no agregado reciclado sobre as características do concreto permeável foram estabelecidas seis composições de agregados, utilizadas na confecção de concretos permeáveis.

Três tipos de resíduos foram utilizados para elaboração das diferentes composições de agregado reciclado. Utilizou-se um RCD proveniente de uma empresa de beneficiamento de resíduos sólidos, cujo teor de cerâmica vermelha era de $2,92 \%$ em massa. Para a elaboração das composições utilizou-se também um resíduo de cerâmica vermelha e um resíduo de concreto, obtidos pela trituração de blocos cerâmicos e corpos de prova de concreto, respectivamente. $\mathrm{O}$ RCD foi misturado com o resíduo de cerâmica na mesma granulometria, adequando os teores a valores pré-definidos (10, 25, 50 e 100\%), conforme a Tabela 1. Como referência, foram utilizados também um agregado convencional (ANAT) e um agregado reciclado sem adição de material cerâmico (AR0).

Tabela 1: Composição dos agregados utilizados na confecção do concreto permeável.

\begin{tabular}{c|l|l}
\hline CONCRETO & AGREGADOS MISTURADOS & COMPOSIÇÃo FINAL \\
\hline ANAT & Agregado convencional & $100 \%$ basalto \\
\hline AR0 & Resíduo de concreto & $100 \%$ resíduo de concreto \\
\hline AR10 & RCD + Resíduo de cerâmica & $10 \%$ cerâmica $+90 \%$ concreto \\
\hline AR25 & RCD + Resíduo de cerâmica & $25 \%$ cerâmica $+75 \%$ concreto \\
\hline AR50 & RCD + Resíduo de cerâmica & $50 \%$ cerâmica $+50 \%$ concreto \\
\hline AR100 & Resíduo de cerâmica & $100 \%$ resíduo de cerâmica \\
\hline
\end{tabular}

Os resultados da caracterização do concreto permeável (coeficiente de permeabilidade, resistência à compressão axial aos 28 dias, índice de volume de vazios, absorção e massa específica) foram analisados estatisticamente com auxílio do software SISVAR [30]. Foi realizada a análise de variância de cada propriedade a um nível de significância de 5\%, seguido pelo Teste de Tukey, com mesmo nível de significância. Os gráficos boxplot foram elaborados por meio do software $\mathrm{R}$.

\subsection{Agregados}

Os agregados utilizados neste estudo foram previamente padronizados em uma distribuição granulométrica uniforme, em que o volume de vazios entre os grãos do agregado é maior [15, 24]. Essa distribuição foi utilizada com o objetivo de atingir uma maior permeabilidade do concreto [23]. A faixa utilizada foi correspondente ao material passante na peneira $12,5 \mathrm{~mm}$ e retido na $9,5 \mathrm{~mm}$.

A composição do RCD foi analisada pelo método de análise visual, conforme a NBR 15116/2004 [11]. O material analisado era composto por 2,92\% de cerâmica vermelha, 95,5\% de fragmentos de rocha e pasta de cimento e $1,58 \%$ de impurezas (vidro, madeira, plástico, material carbonizado).

Foi realizada a caracterização dos agregados graúdos, determinando-se a massa específica e absorção de água de acordo com a NBR NM 53/2009 [31], a massa unitária e o volume de vazios, segundo a NBR NM 45/2006 [32], e o teor de material pulverulento, conforme a NBR NM 46/2003 [33].

A composição química dos agregados foi analisada por fluorescência de raios X, por meio de um espectrômetro Shimadzu, modelo EDX-700. As amostras foram analisadas na forma de pó passante na peneira 
de abertura de 0,075 $\mathrm{mm}$.

\subsection{Concreto permeável}

O traço utilizado foi de 1:5 (cimento: agregado graúdo), em massa, com o objetivo de atingir uma permeabilidade mínima e resistência mínima para pavimentos permeáveis de $20 \mathrm{MPa}$, conforme estabelecido pela NBR 16416/2015 [18] . A relação água/cimento adotada foi de 0,30, correspondente a um valor médio usualmente encontrado na literatura sobre o concreto permeável [13, 15].

Para a confecção do concreto, os agregados foram previamente umedecidos com quantidade de água equivalente à sua absorção, conforme recomendação da ACI 522R [13]. A utilização dos agregados secos pode comprometer a trabalhabilidade da mistura, principalmente para os agregados reciclados com alta absorção de água.

Foram moldados corpos de prova cilíndricos com $100 \mathrm{~mm}$ de diâmetro e $60 \mathrm{~mm}$ de altura, que corresponde à espessura mínima de um pavimento para tráfego de pedestres [18]. Foram utilizados moldes de PVC compatíveis com o permeâmetro utilizado. A mistura do concreto foi realizada de forma mecânica, misturando-se inicialmente os agregados com $50 \%$ da quantidade de água. Na sequência adicionou-se o cimento e a quantidade restante de água, misturando-se até homogeneização do concreto. A moldagem das amostras foi feita com duas camadas compactadas manualmente com a aplicação de 12 golpes de um soquete metálico em cada.

Para cada composição foi realizada a caracterização do concreto permeável conforme as metodologias descritas na Tabela 2 .

Tabela 2: Metodologias utilizadas na caracterização do concreto permeável.

\begin{tabular}{l|c|c}
\hline \multicolumn{1}{c|}{ Propriedade } & № Amostras & Metodologia \\
\hline Massa específica & 6 & ASTM C1754-12 [34] \\
\hline Índice de volume de vazios & 6 & ASTM C1754-12 [34] \\
\hline Absorção de água & 6 & NBR 9778/1987 [35] \\
\hline Coeficiente de permeabilidade & 5 & Adaptado de ACI 522R [13] \\
\hline Resistência à compressão axial & 5 & NBR 9781/2013 [36] \\
\hline
\end{tabular}

O coeficiente de permeabilidade foi determinado por meio de um permeâmetro de carga variável, conforme indicado na ACI 522R [13]. O equipamento foi montado com tubulações e conexões de PVC, de forma similar a outros estudos desenvolvidos nos últimos anos [14, 16, 21, 37], conforme ilustrado na Figura 1.

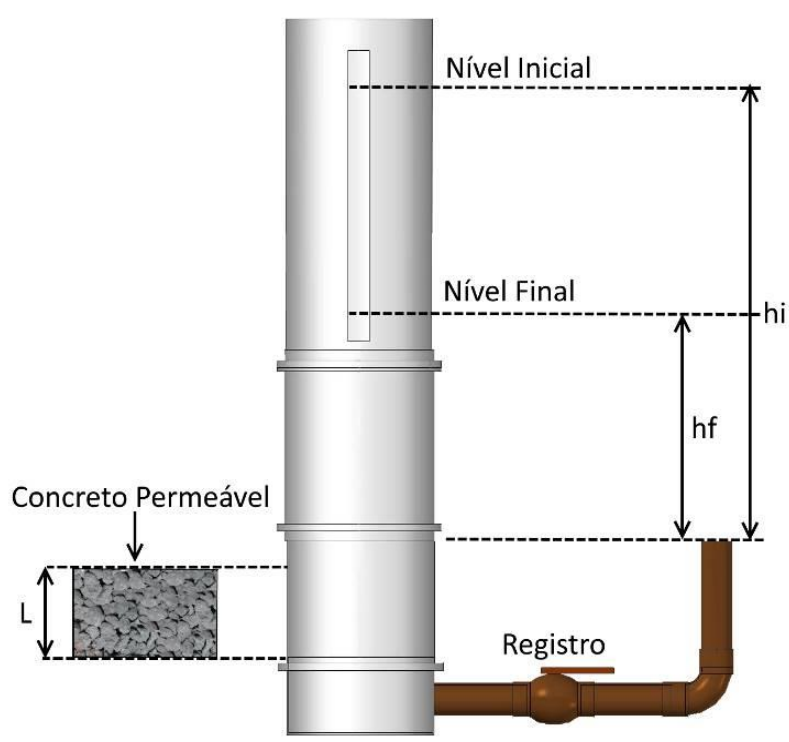

(a)

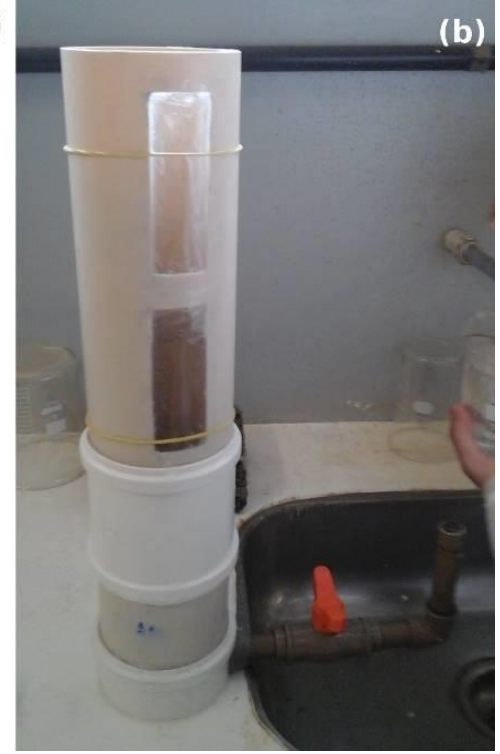

Figura 1: Esquema (a) e foto do permeâmetro (b) de carga variável para determinação do coeficiente de permeabilidade do concreto (onde L é a altura da amostra, hi carga hidráulica inicial e hf carga hidráulica final) 
O coeficiente de permeabilidade foi determinado colocando-se o concreto permeável no equipamento e realizando a saturação do material antes do ensaio. O permeâmetro foi preenchido com água até o nível inicial (hi), distante $30 \mathrm{~cm}$ do nível do extravasor. Abrindo-se o registro de globo, foi medido o tempo necessário ( $\mathrm{t}$ ) para o nível d'água atingir o nível final (hf), $12 \mathrm{~cm}$ acima do extravasor. $\mathrm{O}$ valor do coeficiente de permeabilidade foi calculado por meio da Lei de Darcy, conforme a equação 1.

$$
\mathrm{k}=\frac{\mathrm{A}_{1} \mathrm{~L}}{\mathrm{~A}_{2} \mathrm{t}} \ln \left(\frac{\mathrm{hi}}{\mathrm{hf}}\right)
$$

Onde: $\mathrm{k}$ é o coeficiente de permeabilidade, $\mathrm{A}_{1}$ é a área da seção transversal da amostra, $\mathrm{A}_{2}$ é a área da seção transversal da coluna de carga, L é a altura da amostra, t é o tempo para a água se deslocar do nível inicial hi até o nível final hf, ambos em relação ao nível do extravasor.

\section{RESULTADOS E DISCUSSÕES}

\subsection{Caracterização dos agregados}

Os resultados da caracterização física dos agregados utilizados na confecção dos concretos permeáveis estão expostos na Tabela 3, sendo apresentados na forma de médias.

Tabela 3: Resultados da caracterização física dos agregados natural e reciclados.

\begin{tabular}{|c|c|c|c|c|c|}
\hline Agregado & $\begin{array}{c}\text { Massa Específica } \\
\left({\left.\mathrm{g} . \mathrm{cm}^{-3}\right)}^{-3}\right.\end{array}$ & $\begin{array}{c}\text { Massa Unitária } \\
\left(\mathrm{g} \cdot \mathrm{cm}^{-3}\right) \\
\end{array}$ & $\begin{array}{c}\text { Absorção } \\
(\%)\end{array}$ & $\begin{array}{l}\text { Teor de Material } \\
\text { Pulverulento (\%) }\end{array}$ & $\begin{array}{l}\text { Volume de } \\
\text { Vazios (\%) }\end{array}$ \\
\hline Natural & 3,02 & 1,43 & 0,84 & - & 51,47 \\
\hline Resíduo de Concreto & 2,95 & 1,41 & 4,77 & 0,24 & 46,26 \\
\hline Resíduo de Cerâmica & 2,64 & 0,82 & 22,84 & 2,86 & 50,30 \\
\hline $\mathrm{RCD}$ & 2,62 & 1,17 & 2,18 & 2,73 & 49,83 \\
\hline
\end{tabular}

Nota: O teor de material pulverulento não foi determinado para o agregado natural visto que o material era lavado

Conforme a Tabela 3 todos os agregados apresentaram volume de vazios semelhante, exceto o resíduo de concreto que resultou em um valor inferior aos demais. Os valores são justificados pela utilização de uma mesma distribuição granulométrica (uniforme). Variações no tamanho e forma do agregado podem ter influenciado no volume de vazios do resíduo de concreto.

O resíduo de cerâmica vermelha resultou em uma absorção de água de $22,84 \%$, cerca de 5 vezes o valor de absorção do resíduo de concreto. Esse agregado foi o único que ultrapassou o limite de absorção de água para agregados reciclados, de 12\%, conforme a NBR 15116/2004 [11].

Na Tabela 4 estão apresentados os resultados da caracterização química dos agregados.

Tabela 4: Resultados da caracterização química dos agregados por fluorescência de raios X.

\begin{tabular}{c|c|c|c|c|c|c|c|c|c|c}
\hline \multirow{2}{*}{ Agregado } & \multicolumn{10}{c}{ Composição (\% em massa) } \\
\cline { 2 - 36 } & $\mathrm{SiO}_{2}$ & $\mathrm{Al}_{2} \mathrm{O}_{3}$ & $\mathrm{Fe}_{2} \mathrm{O}_{3}$ & $\mathrm{CaO}$ & $\mathrm{SO}_{3}$ & $\mathrm{TiO}_{2}$ & $\mathrm{~K}_{2} \mathrm{O}$ & $\mathrm{MnO}$ & $\mathrm{ZnO}$ & $\mathrm{SrO}$ \\
\hline Cerâmico & 49,23 & 24,65 & 16,67 & 1,01 & 1,73 & 2,19 & 4,26 & 0,22 & 0,05 & 0,00 \\
\hline Concreto & 30,85 & 8,25 & 20,79 & 32,55 & 1,53 & 3,37 & 1,88 & 0,56 & 0,06 & 0,17 \\
\hline RCD & 39,97 & 7,12 & 8,22 & 37,11 & 1,83 & 1,52 & 3,98 & 0,20 & 0,06 & 0,00 \\
\hline Natural & 34,25 & 11,34 & 28,67 & 17,62 & 1,00 & 4,38 & 2,12 & 0,40 & 0,09 & 0,12 \\
\hline
\end{tabular}

\subsection{Influência do teor de cerâmica nas propriedades do concreto permeável}

Os resultados da caracterização dos seis concretos permeáveis estão expostos na Tabela 5. Os dados estão expressos em valor médio, seguidos da verificação estatística de comparação de médias. 
Tabela 5: Valores médios e análise estatística de comparação de médias dos resultados da caracterização do concreto permeável.

\begin{tabular}{c|c|c|c|c|c|c}
\hline Concreto & $\begin{array}{c}\text { Coeficiente de } \\
\text { Permeabilidade } \\
\left(\mathbf{c m} \cdot \mathbf{s}^{-1}\right)\end{array}$ & $\begin{array}{c}\text { Resistência à } \\
\text { Compressão } \\
\mathbf{( M P a )}\end{array}$ & $\begin{array}{c}\text { Índice de } \\
\text { Volume de } \\
\text { Vazios (\%) }\end{array}$ & $\begin{array}{c}\text { Absorção de } \\
\text { Água (\%) }\end{array}$ & $\begin{array}{c}\text { Massa } \\
\text { Específica } \\
\left(\mathbf{g} \cdot \mathbf{c m}^{-3}\right)\end{array}$ & $\begin{array}{c}\text { Massa Específica } \\
\text { Aparente } \\
\left(\mathbf{g} \cdot \mathbf{c m}^{-3}\right)\end{array}$ \\
\hline ANAT & $0,958 \mathrm{a}$ & $8,84 \mathrm{a}$ & $36,47 \mathrm{a}$ & $4,02 \mathrm{f}$ & $2,94 \mathrm{a}$ & $1,67 \mathrm{a}$ \\
\hline AR0 & $0,932 \mathrm{a}$ & $10,15 \mathrm{a}$ & $32,13 \mathrm{~b}$ & $5,29 \mathrm{e}$ & $2,86 \mathrm{~b}$ & $1,67 \mathrm{a}$ \\
\hline AR10 & $0,879 \mathrm{~b}$ & $2,95 \mathrm{~b}$ & $36,72 \mathrm{a}$ & $8,47 \mathrm{~d}$ & $2,59 \mathrm{e}$ & $1,34 \mathrm{~b}$ \\
\hline AR25 & $0,828 \mathrm{c}$ & $3,20 \mathrm{~b}$ & $37,21 \mathrm{a}$ & $10,59 \mathrm{c}$ & $2,60 \mathrm{de}$ & $1,28 \mathrm{~b}$ \\
\hline AR50 & $0,832 \mathrm{c}$ & $2,66 \mathrm{~b}$ & $37,35 \mathrm{a}$ & $14,89 \mathrm{~b}$ & $2,61 \mathrm{~d}$ & $1,18 \mathrm{c}$ \\
\hline AR100 & $0,819 \mathrm{c}$ & $2,00 \mathrm{~b}$ & $35,13 \mathrm{ab}$ & $25,02 \mathrm{a}$ & $2,63 \mathrm{c}$ & $1,03 \mathrm{~d}$ \\
\hline $\mathrm{N}$ & 5 & 5 & 6 & 6 & 6 & 6 \\
\hline CV $(\%)$ & 2,04 & 20,44 & 6,83 & 3,40 & 0,43 & 3,88 \\
\hline
\end{tabular}

Nota: Médias seguidas de mesma letra na coluna não diferem estatisticamente no Teste de Tukey ao nível de significância de 5\%, sendo N o número de observações e CV o coeficiente de variação.

Na Figura 2 são apresentados os resultados de coeficiente de permeabilidade e resistência à compressão dos concretos permeáveis. Observa-se que os concretos ANAT e AR0 resultaram nos maiores coeficientes de permeabilidade, resultando em 0,958 e $0,932 \mathrm{~cm} \cdot \mathrm{s}^{-1}$, respectivamente. A adição de cerâmica até $10 \%$ em massa ocasionou em uma redução do coeficiente de permeabilidade para $0,879 \mathrm{~cm} \cdot \mathrm{s}^{-1}$. Entre os concretos com teor de material cerâmico superior a $10 \%$, a variação da permeabilidade não foi significativa. É possível inferir sobre uma tendência de redução da permeabilidade com o aumento no teor de material cerâmico no agregado.
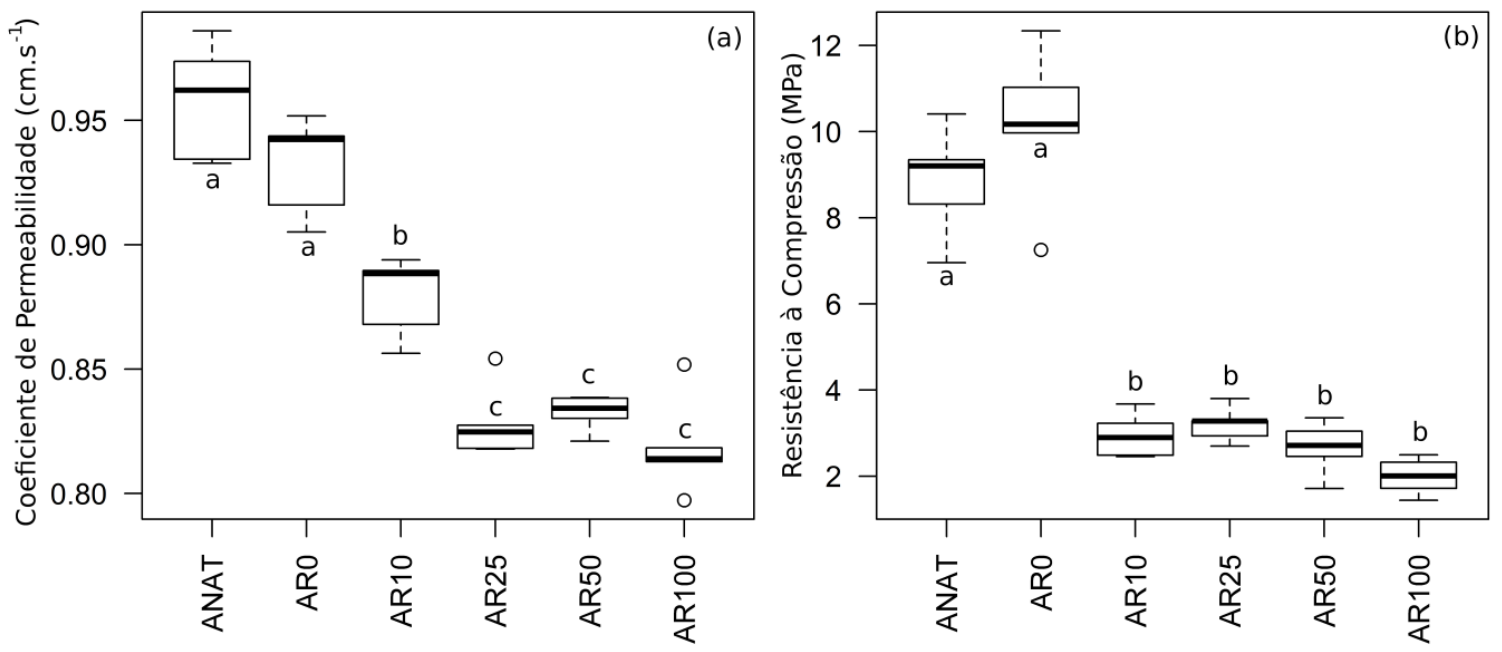

Figura 2: Resultados de coeficiente de permeabilidade (a) e resistência à compressão aos 28 dias (b). Linhas horizontais representam o primeiro, segundo e terceiro quartil. As hastes se estendem até as observações mais distantes, sendo os outliers representados por círculos. Caixas com mesma letra não diferem estatisticamente entre si a um nível de 5\%.

A origem do agregado apresentou influência na permeabilidade dos concretos, sendo este efeito independente da granulometria, uma vez que todos os agregados foram preparados com mesma distribuição granulométrica (uniforme). Resultados similares foram relatados por ĆOSIĆ et al. [22], que indicam que o tipo do agregado possui maior influência na formação dos poros interligados do que a dimensão dos grãos. Outro fator relevante é a absorção do agregado, associado à porosidade dos materiais. O resíduo de cerâmica vermelha apresentou um valor médio de absorção de 22,84\% (Tabela 3), aproximadamente cinco vezes superior à absorção do resíduo de concreto, impactando diretamente na absorção do concreto.

De forma geral, todos os concretos apresentaram um coeficiente de permeabilidade superior a $0,10 \mathrm{~cm} \cdot \mathrm{s}^{-1}$, valor mínimo para considerar um pavimento como permeável [18]. Os valores obtidos foram cerca de 8 a 9 vezes superiores ao valor mínimo, sendo suficientes para a drenagem de águas pluviais pelo 
pavimento.

A maior resistência à compressão axial média obtida foi de $10,15 \mathrm{MPa}$, obtida para o concreto AR0. Verificou-se que embora não tenha ocorrido diferença estatística, o concreto AR0 resultou em resistência média superior ao concreto confeccionado com agregado natural (ANAT). Em todos os concretos contendo material cerâmico a resistência foi inferior a 3,00 MPa. Mesmo a adição de apenas $10 \%$ de material cerâmico ocasionou uma menor resistência do material. Isto pode ser justificado pela menor resistência da cerâmica quando comparado à pasta de cimento, visto que a ruptura dos concretos AR10 a AR100 aconteceu no agregado cerâmico. Já nos concretos ANAT e AR0 a falha ocorreu na interface entre pasta de cimento e agregado.

Os valores de resistência dos concretos ANAT e AR0 foram coerentes com a faixa de 3,5 a $28 \mathrm{MPa}$, sugerida por TENNIS et al. [15]. Entretanto, as resistências dos concretos AR10 a AR100 foram inferiores, refletindo em uma menor qualidade do material para este fim. Na Figura $2 b$ observa-se que não foi detectada diferença estatística entre as resistências dos concretos que continham cerâmica em sua composição (AR10 a AR100).

Nenhum dos concretos analisados atingiu a resistência mínima de $20 \mathrm{MPa}$, estabelecida pela NBR 16416/2015 [18]. Esses concretos não poderiam ser utilizados em pavimentos de trafego de veículos ou estacionamentos, se limitando ao uso em condições de menor solicitação como, por exemplo, passeios e pisos externos.

O aumento da resistência do concreto permeável seria possível por meio da modificação do traço. Maiores quantidades de cimento na mistura proporcionariam um aumento na espessura da pasta de cimento que recobre o agregado, aumentando a resistência do concreto [19]. Na Figura 3a e 3b estão ilustradas duas amostras de concreto que apresentaram fragmentos de cerâmica expostos, demonstrando que não houve uma cobertura eficiente do agregado pela pasta de cimento.

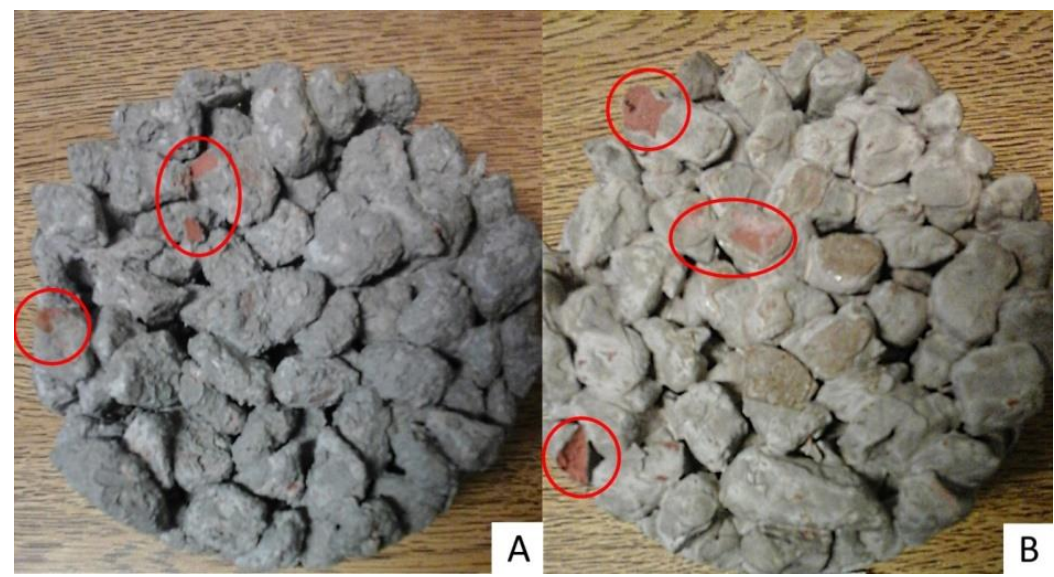

Figura 3: Fragmentos de cerâmica expostos nos concretos AR25 (a) e AR100 (b).

Embora todos os concretos tenham o mesmo traço em massa, a menor massa específica do resíduo de cerâmica e do RCD resultou em uma menor quantidade de pasta de cimento por volume de agregado. Dessa forma, seria necessário aumentar a quantidade de cimento para obter uma resistência superior a $20 \mathrm{MPa}$. Entretanto, o excesso de pasta de cimento ocasiona o preenchimento dos vazios pela pasta de cimento, o que afetaria a permeabilidade do material [13]. Esta discussão ressalta a dificuldade em equacionar o teor de cimento, resistência à compressão e índice de vazios em concretos permeáveis, denotando a importância de aprofundamento do estudo sobre este material.

Pouca variação foi observada no índice de volume de vazios dos concretos permeáveis. O concreto AR0 apresentou o menor índice de volume de vazios, com valor médio de 32,13\%. Os demais materiais não diferiram estatisticamente entre si, resultando em índices de vazios variando de 35,13 a 37,35\%.

A quantidade de vazios no concreto permeável varia de 15 a 35\% [13], sendo recomendados valores entre 15 e $25 \%$ [14]. Volumes de vazios maiores podem comprometer a resistência do concreto [26]. Os valores de índice de vazios superiores a 35\% encontrados neste estudo podem justificar os menores valores de resistência à compressão. Isto é verificado no concreto AR0, que apresentou a maior resistência, porém o menor volume de vazios. Entretanto, nas composições AR10 a AR100 o tipo de agregado teve maior influência na resistência do que o índice de volume de vazios. 
Conforme a Figura 4a, não foi observada uma relação direta entre o teor de material cerâmico no agregado e o índice de volume de vazios. A distribuição granulométrica semelhante dos agregados e os volumes de vazios semelhantes (Tabela 3) contribuíram para que os concretos obtivessem quantidade de vazios próxima. As variações verificadas entre as diferentes composições estão associadas, provavelmente, à compactação do material nos moldes cilíndricos.
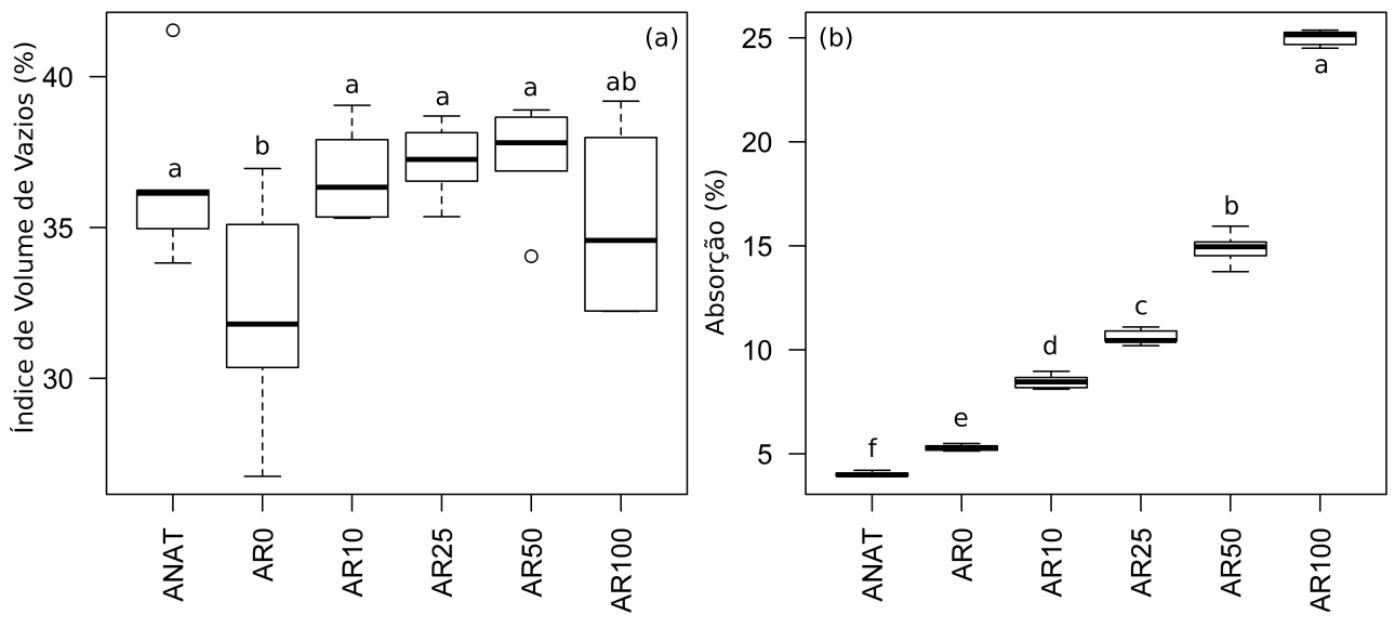

Figura 4: Resultados de índice de volume de vazios (a) e absorção (b) dos concretos permeáveis. Linhas horizontais representam o primeiro, segundo e terceiro quartil. As hastes se estendem até as observações mais distantes, sendo os outliers representados por círculos. Caixas com mesma letra não diferem estatisticamente entre si a um nível de $5 \%$.

A absorção de água dos concretos permeáveis variou de forma proporcional à quantidade de material cerâmico no agregado. $\mathrm{O}$ concreto com agregado natural apresentou menor absorção, com valor médio de $4,02 \%$. O concreto AR0 resultou em uma absorção de água maior que o concreto ANAT, o que pode ser atribuído à maior absorção do resíduo de concreto em relação ao agregado natural. De forma semelhante, devido à maior absorção do resíduo de cerâmica vermelha, a absorção dos concretos AR10 a AR100 foi crescente, conforme ilustrado na Figura 4b.
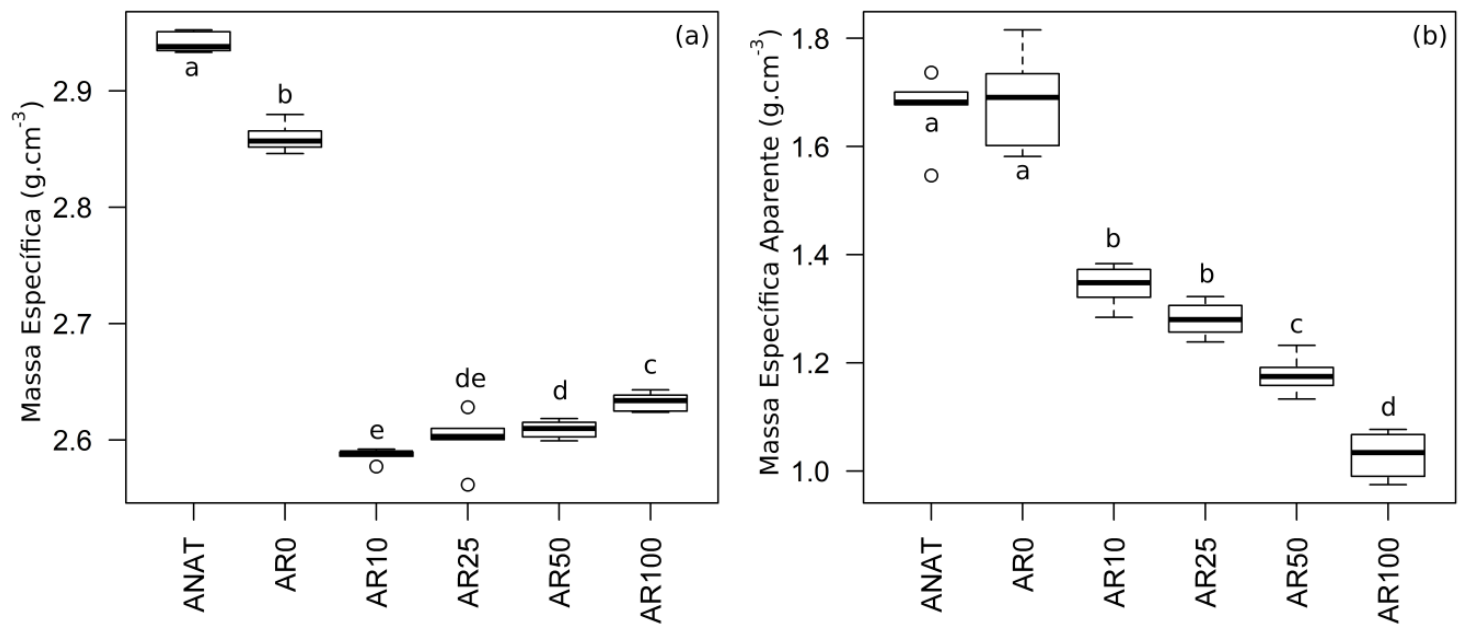

Figura 5: Resultados de massa específica (a) e massa específica aparente (b) dos concretos permeáveis. Linhas horizontais representam o primeiro, segundo e terceiro quartil. As hastes se estendem até as observações mais distantes, sendo os outliers representados por círculos. Caixas com mesma letra não diferem estatisticamente entre si a um nível de 5\%.

A massa específica do concreto permeável apresentou variação coerente com a massa específica dos agregados. O agregado natural, correspondente a maior massa específica, resultou em um concreto com a maior densidade. Conforme a Figura 5a, com o acréscimo de material cerâmico houve uma tendência de aumento na massa específica dos concretos AR10 a AR100. Entretanto, a massa específica do resíduo de cerâmica vermelha e RCD apresentaram diferença inferior a $1 \%$. 
Com relação à massa específica aparente, não houve diferença estatística ente os concretos ANAT e AR0. Neste caso, embora o resíduo de concreto utilizado tenha menor densidade, o seu menor volume de vazios proporcionou um aumento da massa específica do concreto. Os tratamentos que continham material cerâmico apresentaram uma massa específica aparente decrescente conforme a adição do resíduo cerâmico, esse comportamento está ilustrado na Figura 5 b.

O concreto permeável é um material que deve atender simultaneamente requisitos de permeabilidade e resistência. A permeabilidade mínima é estabelecida para que pavimento tenha capacidade de infiltrar a água que precipita durante as chuvas, enquanto que a resistência deve ser suficiente para as solicitações que ocorrerão (tráfego leve, pedestres, veículos estacionados). No entanto, essas duas propriedades usualmente são inversamente proporcionais e dependem principalmente das características dos vazios formados entre os agregados [23].

Diversos estudos realizados recentemente tiveram como objetivo interligar as propriedades do concreto permeável, relacionando principalmente a influência do volume de vazios na resistência e permeabilidade $[13,14,38]$. Na Figura 6 estão expostas as relações entre a resistência à compressão, coeficiente de permeabilidade e o índice de volume de vazios do concreto.
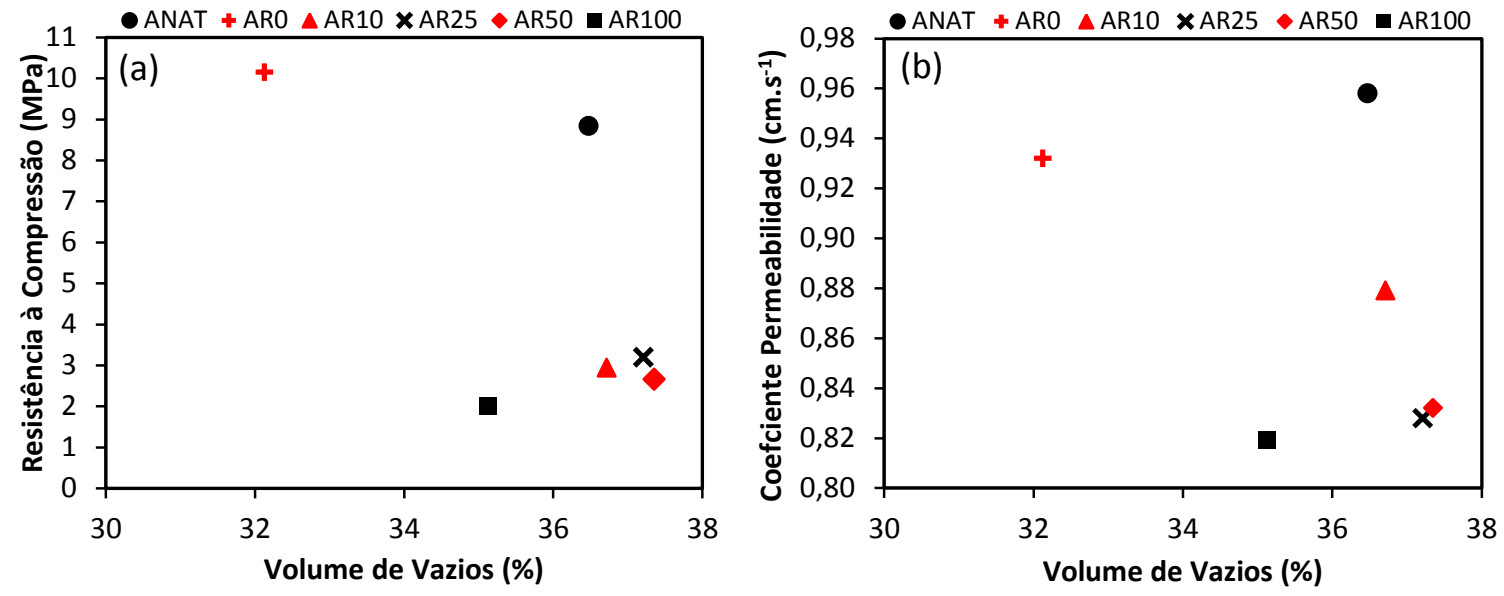

Figura 6: Relação entre a resistência à compressão (a), coeficiente de permeabilidade (b) e o índice de volume de vazios.

O índice de volume de vazios das amostras não variou significativamente, com exceção do concreto AR0. Conforme a Figura $6 \mathrm{a}$ e 6 b, não foi possível estabelecer uma relação da influência do volume de vazios na resistência à compressão e no coeficiente de permeabilidade do concreto. Entretanto, devido à utilização de diferentes composições do agregado, foram detectadas diferenças estatísticas na resistência à compressão e no coeficiente de permeabilidade. Observou-se que a resistência à compressão e o coeficiente de permeabilidade do concreto apresentaram influência da massa específica aparente e da absorção de água, respectivamente. As relações entre os valores médios das propriedades descritas estão ilustradas na Figura 7.
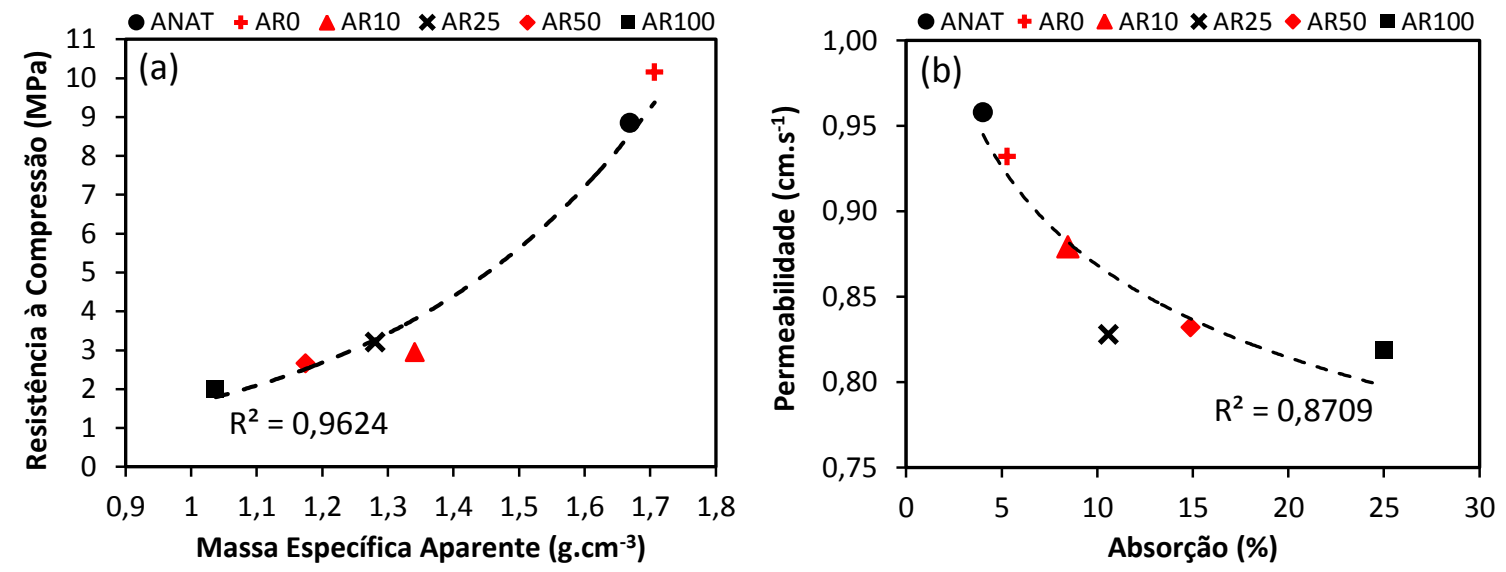

Figura 7: Relação entre a massa específica aparente e resistência (a) e entre a permeabilidade e absorção de água (b). 
É possível verificar que a resistência à compressão foi crescente em função do aumento da massa específica aparente. A maior massa específica em conjunto com o menor volume de vazios do concreto AR0 pode ter contribuído para o mesmo resultar em resistência superior ao concreto com agregado natural. O coeficiente de permeabilidade do concreto reduziu com o aumento da absorção de água, com exceção do tratamento AR25, que pode ter sido influenciado pela compactação do material ou a interação entre os dois agregados misturados (RCD e cerâmica) para esta proporção. A absorção de água não deveria influenciar no coeficiente de permeabilidade, visto que antes da utilização do permeâmetro os corpos de prova estavam na condição saturada. A causa provável para a interferência é que as características geométricas de cada agregado, como tamanho e forma, tenham influenciado na formação dos vazios interligados [27]. Além disso, a prémolhagem dos agregados conforme a recomendação do ACI 522R [13] pode ter influenciado no preenchimento dos vazios pela pasta de cimento, principalmente para o agregado cerâmico que necessitou de maior quantidade de água devido a sua absorção.

\section{CONCLUSÕES}

Os resultados obtidos indicam que o uso de agregados reciclados mistos em substituição dos agregados naturais altera significativamente as propriedades do concreto permeável. $\mathrm{O}$ teor de material cerâmico do agregado reciclado também tem influência nas características do material, podendo ser um fator limitante.

A utilização de agregados com distribuição granulométrica uniforme e traço 1:5 proporcionou permeabilidades superiores ao valor mínimo estipulado pela NBR 16416/2015 [18], de $0,10 \mathrm{~cm} \cdot \mathrm{s}^{-1}$. O coeficiente de permeabilidade reduziu com o aumento de material cerâmico, que pode ser associado à composição do agregado visto que os volumes de vazios não diferiram estatisticamente.

O traço utilizado não possuiu quantidade de cimento suficiente para que a resistência do concreto atinja $20 \mathrm{MPa}$. Isso limitaria a utilização desse concreto a elementos com menor solicitação, como pisos externos e calçadas. Para o emprego do material em pavimentos e estacionamentos seria necessária a reformulação do traço, adequando a resistência ao valor mínimo de $20 \mathrm{MPa}$.

A utilização de resíduo de concreto como agregado resultou em resistência similar ao concreto confeccionado com agregado natural. Os agregados com material cerâmico, no entanto, reduziram significativamente a resistência, mesmo para um teor de $10 \%$. Nesse caso a resistência da cerâmica foi determinante para a resistência do concreto.

O índice de volume de vazios não apresentou relação direta com o coeficiente de permeabilidade ou a resistência à compressão, provavelmente devido às diferenças de composição dos agregados utilizados em cada mistura.

Mesmo em quantidades pequenas, o material cerâmico foi prejudicial ao concreto permeável, reduzindo tanto a sua resistência como permeabilidade. O concreto confeccionado com resíduo de concreto apresentou características semelhantes ao concreto de agregado natural. Assim, há possibilidade de sua utilização como material alternativo ao agregado convencional.

\section{AGRADECIMENTOS}

Os autores agradecem ao Programa de Pós-Graduação em Engenharia Sanitária e Ambiental e ao Laboratório de Engenharia de Materiais da Universidade Estadual de Ponta Grossa (UEPG) pelo apoio na elaboração deste estudo.

\section{BIBLIOGRAFIA}

[1] DAHLBO, H., BACHÉR, J., LÄHTINEN, K., et al., "Construction and demolition waste management a holistic evaluation of environmental performance", Journal of Cleaner Production, v. 107, pp. 333-341, Nov. 2015.

[2] BEHERA, M., BHATTACHARYYA, S. K., MINOCHA, A. K., et al., "Recycled aggregate from C\&D waste $\&$ its use in concrete - A breakthrough towards sustainability in construction sector: A review", Construction and Building Materials, v. 68, pp. 501-516, Out. 2014.

[3] VIEIRA, C. S., PEREIRA, P. M., "Use of recycled construction and demolition materials in geotechnical applications: A review", Resources, Conservation and Recycling, v. 103, pp. 192-204, Out. 2015.

[4] RODRÍGUEZ, G., MEDINA, C., ALEGRE, F. J., et al., "Assessment of Construction and Demolition Waste plant management in Spain: in pursuit of sustainability and eco-efficiency", Journal of Cleaner Production, v. 90, pp. 16-24, Mar. 2015. 
[5] FROTTÉ, C., NUBILA, C. S. A. Di, NAGALLI, A., et al., "Estudo das propriedades físicas e mecânicas de concreto com substituição parcial de agregado natural por agregado reciclado proveniente de RCD", $M a$ téria, v. 22, n. 2, e11811, Jun. 2017.

[6] SANTOS, D. O. J., FONTES, C. M. A., LIMA, P. R. L., "Uso de agregado miúdo reciclado em matrizes cimentícias para compósitos reforçados com fibras de sisal", Matéria, v. 22, n. 1, e11801, Mai. 2017.

[7] ASSOCIAÇÃO BRASILEIRA DE EMPRESAS DE LIMPEZA PÚBLICA E RESÍDUOS ESPECIAIS, Panorama dos resíduos sólidos no Brasil, São Paulo, ABRELPE, 2014.

[8] BRASIL. CONSELHO NACIONAL DO MEIO AMBIENTE, Resolução ${ }^{\circ} 307$ de 5 de Julho de 2002, Estabelece diretrizes, critérios e procedimentos para a gestão dos resíduos da construção civil, Diário Oficial da União, Brasília, Jul. 2002.

[9] ANGULO, S. C., TEIXEIRA, C. E., CASTRO, A. L., et al., "Resíduos de construção e demolição: avaliação de métodos de quantificação", Ambiente Construído, v. 13, n. 2, pp. 61-73, Abr./Jun. 2013.

[10] ASSOCIAÇÃO BRASILEIRA PARA RECICLAGEM DE RESÍDUOS DA CONSTRUÇÃO CIVIL E DEMOLIÇÃO, Pesquisa setorial de reciclagem de resíduos da construção, ABRECON, 2015.

[11] ASSOCIAÇÃO BRASILEIRA DE NORMAS TÉCNICAS. NBR 15.116: Agregados reciclados de resíduos sólidos da construção civil - Utilização em pavimentação e preparo de concreto sem função estrutural - Requisitos, Rio de Janeiro, 2004.

[12] CHANDRAPPA, A., BILIGIRI, K., "Pervious concrete as a sustainable pavement material - Research findings and future prospects: A state-of-art review", Construction and Building Materials, v. 111, pp. 262274, Mai. 2016.

[13] AMERICAN CONCRETE INSTITUTE. ACI 522R-10: Report on pervious concrete, Farmington Hills, 2010.

[14] IBRAHIM, A., MAHMOUD, E., YAMIN, M., et al., "Experimental study on Portland cement pervious concrete mechanical and hydrological properties", Construction and Building Materials, v. 50, pp. 524-529, Jan. 2014

[15] TENNIS, P. D., LEMING, M. L., AKERS, D. J., Pervious Concrete Pavements, Skokie, Portland Cement Association, 2004.

[16] NEITHALATH, N., SUMANASOORIYA, M. S., DEO, O., "Characterizing pore volume, sizes, and connectivity in pervious concretes for permeability prediction", Materials Characterization, v. 61, n. 8, pp. 802-813, Ago. 2010.

[17] BHUTTA, M. A. R., HASANAH, N., HUSSIN, M. W., et al., "Properties of porous concrete from waste crushed concrete (recycled aggregate)", Construction and Building Materials, v. 47, n. 8, pp. 12431248, Out. 2013.

[18] ASSOCIAÇÃO BRASILEIRA DE NORMAS TÉCNICAS. NBR 16416: Pavimentos permeáveis de concreto - Requisitos e procedimentos, Rio de Janeiro, 2015.

[19] TORRES, A., HU, J., RAMOS, A., "The effect of the cementitious paste thickness on the performance of pervious concrete", Construction and Building Materials, v. 95, pp. 850-859, Out. 2015.

[20] ZAETANG, Y., SATA, Y., WONGSA, A., CHINDAPRASIRT, P., "Properties of pervious concrete containing recycled concrete block aggregate and recycled concrete aggregate", Construction and Building Materials, v. 111, pp. 15-21, Mai. 2016.

[21] CHANDRAPPA, A. K., BILIGIRI, K. P., "Comprehensive investigation of permeability characteristics of pervious concrete: A hydrodynamic approach", Construction and Building Materials, v. 123, pp. 627-637, Out. 2016.

[22] ĆOSIĆ, K., KORAT, L., DUCMAN, V., et al., "Influence of aggregate type and size on properties of pervious concrete", Construction and Building Materials, v. 78, pp. 69-76, Mar. 2015.

[23] JOSHAGHANI, A., RAMEZANIANPOUR, A. A., JABERIZADEH, M., "Mechanical characteristic of pervious concrete considering the gradation and size of coarse aggregates", Research Journal of Environmental and Earth Sciences, v. 6, n. 9, pp. 437-442, Set. 2014.

[24] CROUCH, L. K., PITT, J., HEWITT, R., "Aggregate effects on pervious Portland cement concrete static modulus of elasticity", Journal of Materials in Civil Engineering, v. 19, n. 7, pp. 561-568, Jul. 2007.

[25] PUTMAN, B., NEPTUNE, A., "Comparison of test specimen preparation techniques for pervious concrete pavements", Construction and Building Materials, v. 25, n. 8, pp. 3480-3485, Ago. 2011. 
[26] HUANG, B., WU, H., SHU, X., et al., "Laboratory evaluation of permeability and strength of polymermodified pervious concrete", Construction and Building Materials, v. 24, pp. 818-823, Mai. 2010.

[27] DEO, O., NEITHALATH, N., "Compressive behavior of pervious concretes and a quantification of the influence of random pore structure features", Materials Science and Engineering A, v. 528, n. 1, pp. 402-412, Nov. 2010.

[28] TAVARES, L. M., KAZMIERCZAK, C. S., "Estudo da influência dos agregados de concreto reciclado em concretos permeáveis", Revista IBRACON de Estruturas, v. 9, n. 1, pp. 75-90, Fev. 2016.

[29] ANGULO, S. C., JOHN, V. M., ULSEN, C., et al., "Separação óptica do material cerâmico dos agregados mistos de resíduos de construção e demolição", Engenharia Sanitária e Ambiental, v. 16, n. 3, pp. 299306, Jul./Set. 2013.

[30] FERREIRA, D.F. Sistema de análise de variância, Versão 5.6, Lavras, UFLA, 2015.

[31] ASSOCIAÇÃO BRASILEIRA DE NORMAS TÉCNICAS. NBR NM 53: Agregado graúdo - Determinação de massa específica, massa específica aparente e absorção de água, Rio de Janeiro, 2009.

[32] ASSOCIAÇÃO BRASILEIRA DE NORMAS TÉCNICAS. NBR NM 45: Agregados - Determinação da massa unitária e do volume de vazios, Rio de Janeiro, 2006.

[33] ASSOCIAÇÃO BRASILEIRA DE NORMAS TÉCNICAS. NBR NM 46: Agregados - Determinação do material fino que passa através da peneira $75 \mu \mathrm{m}$, por lavagem, Rio de Janeiro, 2003.

[34] AMERICAN SOCIETY FOR TESTING AND MATERIALS. ASTM C-1754: Standard test method for density and void content of hardened pervious concrete, West Conshohocken, 2012.

[35] ASSOCIAÇÃO BRASILEIRA DE NORMAS TÉCNICAS. NBR 9778: Argamassa e concreto endurecidos - Determinação da absorção de água por imersão - Índice de vazios e massa específica, Rio de Janeiro, 1987.

[36] ASSOCIAÇÃO BRASILEIRA DE NORMAS TÉCNICAS. NBR 9781: Peças de concreto para pavimentação - Especificação e métodos de ensaio, Rio de Janeiro, 2013.

[37] NGUYEN, D. H., SEBAIBI, N., BOUTOUIL, M., et al., "A modified method for the design of pervious concrete mix", Construction and Building Materials, v. 73, pp. 271-282, Set. 2014.

[38] LIAN, C., ZHUGE, Y., BEECHAM, S., "The relationship between porosity ans strength for porous concrete", Construction and Building Materials, v. 25, n. 11, pp. 4294-4298, Nov. 2011. 